\title{
A sticky situation: extracellular DNA shapes Aspergillus fumigatus biofilms
}

\author{
Sven Krappmann ${ }^{1 *}$ and Gordon Ramage ${ }^{2 *}$ \\ Microbiology Institute - Clinical Microbiology, Immunology and Hygiene, University Hospital of Erlangen, Friedrich-Alexander-University Erlangen-Nürnberg, \\ Erlangen, Germany \\ ${ }^{2}$ School of Medicine, College of Medical, Veterinary and Life Sciences, University of Glasgow, Glasgow, UK \\ *Correspondence: sven.krappmann@uk-erlangen.de; gordon.ramage@glasgow.ac.uk
}

Edited by:

Helio K. Takahashi, Universidade Federal de Sao Paulo, Brazil

Reviewed by:

Helio K. Takahashi, Universidade Federal de Sao Paulo, Brazil

\section{A commentary on}

Extrinsic extracellular DNA leads to biofilm formation and colocalizes with matrix polysaccharides in the human pathogenic fungus Aspergillus fumigatus by Shopova, I., Bruns, S., Thywissen, A., Kniemeyer, O., Brakhage, A. A., and Hillmann, F. (2013). Front. Microbiol. 4:141. doi: 10.3389/fmicb.2013.00141

Multicellular stages play an important role for microbial communities as well as during infections. For one of the major fungal pathogens, the environmental mold Aspergillus fumigatus, an interesting and relevant morphotype beyond the conidial and hyphal stages has gained increasing attention: the multicellular biofilm. Such hyphal communities were first described for A. fumigatus in 2007 (Mowat et al., 2007) and are nowadays recognized to be relevant in the clinic (Müller et al., 2011).

A. fumigatus is environmentally omnipresent and its airborne conidia are constantly inhaled to become eliminated by the functional host immune system. In case of immune response failure, these infectious propagules germinate and invade the infected tissue to cause forms of aspergillosis (Dagenais and Keller, 2009). Treatment options for this disease are still inadequate, based on the limited number of antifungal drugs and their side effects. Resistance of $A$. fumigatus to antifungal treatment is influenced by several factors, among them biofilm formation (Seidler et al., 2008). In these structures, a dense network of hyphae becomes embedded in an extracellular matrix (ECM) that is mainly composed of polysaccharides.
The ECM of such biofilms grown in vitro further contains melanin, proteins like hydrophobins or antigens, polyols, and monosaccharides (Beauvais et al., 2007). A. fumigatus biofilms are also formed in vivo during invasive aspergillosis or aspergilloma and have been characterized to slightly differ from their in vitro counterparts with respect to ECM composition (Loussert et al., 2010).

Recent evidence has identified extracellular DNA (eDNA) as important component of A. fumigatus biofilms, either derived from fungal autolysis (Rajendran et al., 2013) or, as elegantly demonstrated in a recent publication by Brakhage, Hillmann, and colleagues, when externally supplied (Shopova et al., 2013). Probably supported by a high affinity of nucleic acids to the cell wall of $A$. fumigatus (Jöchl et al., 2009) that is based on electrostatic interactions, extrinsic eDNA promotes adhesion of the conidia in the initial phase of biofilm formation, triggers polysaccharide formation and becomes incorporated in the biofilm, thereby shaping its overall structure. Accordingly, DNA contributes to the structural integrity of mature A. fumigatus biofilms, a finding that has been described for several bacterial biofilm formers before (Flemming and Wingender, 2010) and that highlights the structural conservation of microbial biofilms. Considering that A. fumigatus commonly colonizes patients suffering from cystic fibrosis and that the mucus of such patients contains extrinsic DNA at high concentrations that may stem from of neutrophil extracellular traps (NETs) (Brinkmann et al., 2004; Bruns et al., 2010), which are released by a special form of cell death termed NETosis (Remijsen et al., 2011; Almyroudis et al., 2013), from necrotic tissue, or from competing microorganisms, the formation of biofilms is of special significance in this particular host group.

In the light of the clinical as well as industrial relevance of Aspergillus biofilms (Ramage et al., 2011), the newly presented data and insights not only sharpen our image of this multicellular stage within the Aspergillus lifestyle but also provide perspective for improvement and new avenues in antifungal therapy or biotechnological applications.

\section{REFERENCES}

Almyroudis, N. G., Grimm, M. J., Davidson, B. A., Röhm, M., Urban, C. F., and Segal, B. H. (2013). NETosis and NADPH oxidase: at the intersection of host defense, inflammation, and injury. Front. Immunol. 4:45. doi: 10.3389/fimmu.2013.00045

Beauvais, A., Schmidt, C., Guadagnini, S., Roux, P., Perret, E., Henry, C., et al. (2007). An extracellular matrix glues together the aerialgrown hyphae of Aspergillus fumigatus. Cell. Microbiol. 9, 1588-1600. doi: 10.1111/j.1462 5822.2007.00895.x

Brinkmann, V., Reichard, U., Goosmann, C., Fauler, B., Uhlemann, Y., Weiss, D. S., et al. (2004). Neutrophil extracellular traps kill bacteria. Science 303, 1532-1535. doi: 10.1126/science. 1092385

Bruns, S., Kniemeyer, O., Hasenberg, M., Aimanianda, V., Nietzsche, S., Thywissen, A., et al. (2010). Production of extracellular traps against Aspergillus fumigatus in vitro and in infected lung tissue is dependent on invading neutrophils and influenced by hydrophobin RodA. PLoS Pathog. 6:e1000873. doi: 10.1371/journal.ppat.1000873

Dagenais, T. R., and Keller, N. P. (2009). Pathogenesis of Aspergillus fumigatus in invasive Aspergillosis. Clin. Microbiol. Rev. 22, 447-465. doi: 10.1128/CMR.00055-08

Flemming, H. C., and Wingender, J. (2010). The biofilm matrix. Nat. Rev. Microbiol. 8, 623-633. doi: $10.1038 /$ nrmicro2415 
Jöchl, C., Loh, E., Ploner, A., Haas, H., and Hüttenhofer, A. (2009). Development-dependent scavenging of nucleic acids in the filamentous fungus Aspergillus fumigatus. RNA Biol. 6, 179-186. doi: 10.4161/rna.6.2.7717

Loussert, C., Schmitt, C., Prevost, M. C., Balloy, V., Fadel, E., Philippe, B., et al. (2010). In vivo biofilm composition of Aspergillus fumigatus. Cell. Microbiol. 12, 405-410. doi: 10.1111/j.14625822.2009.01409.x

Mowat, E., Butcher, J., Lang, S., Williams, C., and Ramage, G. (2007). Development of a simple model for studying the effects of antifungal agents on multicellular communities of Aspergillus fumigatus. J. Med. Microbiol. 56, 1205-1212. doi: 10.1099/jmm.0.47247-0

Müller, F. M., Seidler, M., and Beauvais, A. (2011). Aspergillus fumigatus biofilms in the clinical setting. Med. Mycol. 49(Suppl. 1), S96-S100. doi: 10.3109/13693786.2010.502190

Rajendran, R., Williams, C., Lappin, D. F., Millington, O., Martins, M., and Ramage, G. (2013). Extracellular DNA release acts as an antifungal resistance mechanism in mature Aspergillus fumigatus biofilms. Eukaryot. Cell 12, 420-429. doi: 10.1128/EC.00287-12

Ramage, G., Rajendran, R., Gutierrez-Correa, M., Jones, B., and Williams, C. (2011). Aspergillus biofilms: clinical and industrial significance. FEMS Microbiol. Lett. 324, 89-97. doi: 10.1111/j.1574-6968.2011. 02381.x

Remijsen, Q., Kuijpers, T. W., Wirawan, E., Lippens, S., Vandenabeele, P., and Vanden Berghe, T. (2011). Dying for a cause: NETosis, mechanisms behind an antimicrobial cell death modality. Cell Death Differ. 18, 581-588. doi: 10.1038/cdd. 2011.1

Seidler, M. J., Salvenmoser, S., and Müller, F. M. (2008). Aspergillus fumigatus forms biofilms with reduced antifungal drug susceptibility on bronchial epithelial cells. Antimicrob. Agents Chemother. 52, 4130-4136. doi: 10.1128/AAC.00234-08

Shopova, I., Bruns, S., Thywissen, A., Kniemeyer, O., Brakhage, A. A., and Hillmann, F. (2013).
Extrinsic extracellular DNA leads to biofilm formation and colocalizes with matrix polysaccharides in the human pathogenic fungus Aspergillus fumigatus. Front. Microbiol. 4:141. doi: $10.3389 /$ fmicb. 2013.00141

Received: 24 May 2013; accepted: 31 May 2013; published online: 28 June 2013.

Citation: Krappmann S and Ramage G (2013) A sticky situation: extracellular DNA shapes Aspergillus fumigatus biofilms. Front. Microbiol. 4:159. doi: 10.3389/ fmicb.2013.00159

This article was submitted to Frontiers in Fungi and Their Interactions, a specialty of Frontiers in Microbiology.

Copyright (c) 2013 Krappmann and Ramage. This is an open-access article distributed under the terms of the Creative Commons Attribution License, which permits use, distribution and reproduction in other forums, provided the original authors and source are credited and subject to any copyright notices concerning any third-party graphics etc. 